\title{
Ceramic Total Knee Arthroplasty: Ready to Go?
}

\author{
G. Solarino ${ }^{1}$ \\ C. Piconi ${ }^{2}$ \\ V. De Santis ${ }^{3}$ \\ A. Piazzolla ${ }^{1}$ \\ 1 Orthopaedics Unit, Department of Basic Medical Sciences, \\ Neurosciences and Sensory Organs, University of Bari “Aldo Moro," \\ Bari, Italy \\ 2 National Research Council, Institute for the Science and Technology \\ of Ceramic Materials (ISTEC-CNR), Faenza, Ravenna, Italy \\ ${ }^{3}$ Department of Orthopedics and Traumatology, Catholic University, \\ Rome, Italy
}

B. Moretti ${ }^{1}$

Joints 2017;5:224-228.

\begin{abstract}
Address for correspondence G. Solarino, MD, Orthopaedics Unit, Department of Basic Medical Sciences, Neurosciences and Sensory Organs, University of Bari “Aldo Moro," Piazza Giulio Cesare 11, Bari 70124, Italy (e-mail: giuseppe.solarino@uniba.it).
\end{abstract}

\begin{abstract}
Keywords

- knee replacement

- arthroplasty

- ceramic composite

- metal

- hypersensitivity

Total knee arthroplasty (TKA) is a well-established surgical procedure in the late stages of knee osteoarthritis. Nevertheless, this procedure is associated with a percentage of unsatisfactory results and biomechanical failures, with aseptic loosening being the most common cause of revision. Beside these problems, cutaneous and systemic hypersensitivity reactions to metals have arisen as an increasing concern after joint arthroplasties, even if allergies against implant materials are still a quite rare and not well-known problem. Ceramic composites have been recently used in prosthetic components, showing minimum wear and excellent long-term results in total hip replacement, due to their high resistance to scratching and their better wettability with respect to cobalt-chromium alloy. Furthermore, the biologic response to debris generated from these bearings is less aggressive. Knee joint simulator tests and clinical results demonstrate promising results of TKAs with ceramic components that should led to benefit for the patients.
\end{abstract}

\section{Introduction}

Total knee arthroplasty (TKA) is currently considered the treatment of choice in patients suffering from osteoarthritis $(\mathrm{OA})$ of the knee when therapies to reduce joint pain have no more effects. This surgical procedure has proven to be very successful. The third report of the Italian Arthroplasty Registry Project ${ }^{1}$ showed that more than 67,000 primary TKAs were implanted in 2014, and the growing rate from 2001 is $7 \%$ per year. However, the success of this procedure is accompanied by complications. The Emilia-Romagna Registry of Orthopedic Prosthetic Implants showed that $55.7 \%$ of the revision TKAs performed between 2000 and 2013 were due to aseptic loosening of the implant or of the tibial or femoral component only. $^{2}$

Several factors have been proposed to explain the cause of mechanical failure and patient's unsatisfaction: incorrect limb alignment, ligamentous laxity and joint instability, poor fixation, and limited range of motion. Nevertheless, several investigations have been performed to compare different sur- gical and technical approaches with the aim to identify the drawbacks of the procedure and minimize unsuccessful outcomes. There is no strong evidence on several efficacy issues that remain under debate, such as posterior-stabilized versus cruciate-retaining implants, high-flexion design of femoral components, replacing of the patella (yes or not), and fixedversus mobile-bearing tibial surface. ${ }^{3-7}$

Recently, new materials and solutions have been introduced to limit wear of prosthetic joints, such as highly crosslinked and antioxidant-doped polyethylenes for the tibial insert, and ceramicized femoral and tibial components. ${ }^{8,9}$ Efficacy of ceramic components in decreasing polyethylene wear was demonstrated since the early alumina TKAs and was confirmed by more recent designs. ${ }^{10}$

Besides biomechanical problems, some concerns exist about the risk of developing cutaneous and systemic hypersensitivity reactions to metals forming the alloys used in implantable devices. Hypersensitivity reactions is still an unknown problem that can occur after joint replacement; symptoms such as pain, effusion, pruritus, erythema, and/or published online October 26, 2017
DOI https://doi.org/ 10.1055/s-0037-1607428. ISSN 2282-4324.
Copyright (c) 2017 Georg Thieme Verlag License terms KG Stuttgart · New York

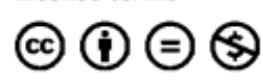


decreased mobility are often aspecific, but this complication should be taken into consideration when low-grade infection and other mechanical problems have been excluded. ${ }^{11}$

Although rare, allergy to metallic components can lead to revision surgery because conservative treatment is unresponsive. Ions released by corrosion of metallic wear debris may play a critical role and metal particles can be found in the soft tissues surrounding the implant. Kretzer et al observed that during a standard knee wear test, approximately $12 \%$ by weight of the wear products were metallic and postulated that particles and ions may become clinically relevant for sensitive patients. ${ }^{12}$

According to the 2016 Australian Arthroplasty Register, ${ }^{13}$ approximately $2 \%$ of revision TKAs are attributed to "metalrelated pathology" and a legal action-as a result of failed implants due to perceived allergy-has been instigated in the United States against a TKA manufacturer. According to Middleton and Toms, ${ }^{14}$ patients with a preexisting hypersensitivity will always believe any problems they may have after their knee replacement are related to "metallic allergy."

Indeed, immunological reactive mechanism to metallic components is still an unclear and debated issue, and moreover its clinical evidence is increasing. Consequently, several manufacturing companies are marketing actively hypoallergenic implants to be used specifically in sensitive patients. Ajwani and Charalambous ${ }^{15}$ contacted and questioned 22 companies from the United Kingdom National Joint Registry to obtain information about availability and characteristics (designs and materials) of their implants. Replies were obtained from 13 manufacturers, providing information on 23 different models of TKA. Among these, 15 systems had a "hypersensitivity-friendly" option both for tibial and femoral components which was identical in terms of design and instrumentation to the conventional system.

The use of ceramic components in TKA results in an interesting option. Ceramic oxides (alumina and zirconia) are in clinical use for a long time, and the absence of local and systemic reactions to ceramic wear debris is a well-assessed behavior, ${ }^{16}$ as well as their improved mechanical properties in comparison with alumina (-Table $\mathbf{1}$ ). In addition, scratching resistance of ceramic composites is much higher than that of bare or ceramicized metals. Load to scratch measured for BIOLOXdelta (CeramTec, Plochingen, Germany) is about 5-fold that of oxidized zirconium alloy surface and about 10 -fold that of CoCrMo alloy, ${ }^{17}$ thus resulting in a lower damage by third bodies of the

Table 1 Comparison of selected properties between contemporary medical grade alumina and AMC

\begin{tabular}{|l|l|l|l|}
\hline Property & Unit & Alumina & AMC \\
\hline Density & $\mathrm{g} / \mathrm{cm}^{3}$ & $3.96-3.98$ & 4.37 \\
\hline $\begin{array}{l}\text { Bending } \\
\text { strength }\end{array}$ & $\mathrm{MPa}$ & $550-630$ & $>1,350$ \\
\hline $\begin{array}{l}\text { Fracture } \\
\text { toughness }\end{array}$ & $\mathrm{MPa} \mathrm{\textrm {m } ^ { 1 / 2 }}$ & $3.5-4$ & 7 \\
\hline Hardness & $\mathrm{GPa}$ & $19-20$ & 17.5 \\
\hline
\end{tabular}

Abbreviation: AMC, alumina-matrix composite. articulating surfaces and in a lower wear of the polyethylene inserts.

\section{The Development of Ceramic TKAs}

The first "ceramic" knee arthroplasty was implanted in 1890 in Berlin by Gluck. ${ }^{18}$ He conceived an hinged device made up of ivory, a natural calcium phosphate ceramic that was fixed on the tibia and femur with a mixture of colophonia and plaster of Paris.

The first modern ceramic knee arthroplasty was used in 1972 by Langer at the University of Jena in Germany. ${ }^{19}$ The implant (KWH, Hermsdorf, Germany) was made of polycrystalline alumina and replaced only partially the tibial plateau. Implant was uncemented and stability was achieved with the help of a network of perpendicularly crossing grooves on the side of the implant facing the host bone, while the side in contact with the femoral condyle was polished and slightly concave. Partial tibia plateau replacements with this implant were performed in 72 patients ranging in age from 23 to 79 years. Clinical outcomes 5 years postoperatively were reported as good. ${ }^{20}$

Recently, ceramic TKA designs were developed by Japanese researchers and manufactured by Kyocera Company (Kyoto, Japan). The common features of those implants were the use of alumina for femoral and tibial components and ceramicpolyethylene articular couples. Cruciate-retaining and posterior-stabilized designs were both available and ceramic patellar component as well. This kind of implants was first reported by Oonishi et al, ${ }^{21}$ who used the KOM 1 cementless implant in 134 patients. Inoue et $\mathrm{al}^{22}$ reported early results on another alumina total knee system with a polyethylene insert (KC-1; Kyoto Ceramics Company, now Kyocera). The clinical use of these early devices with alumina components led to a dramatic decrease in polyethylene wearing, likely due to the better finish and wettability, and resistance to scratching of alumina with respect to $\mathrm{CoCr}$ alloy.

Cementless designs were acknowledged soon as the cause of some failures, and the design changed consequently. ${ }^{23}$ Moreover, limited flexural resistance of alumina implied a bulky design of the implant components, and then the design was improved using yttria-stabilized zirconia (Y-TZP) for the femoral component and $\mathrm{Ti}$ alloy or $\mathrm{CoCr}$ alloy for the tibial tray, both cemented. Zirconia was very interesting for the production of the femoral component in TKA because it could be made almost in the same size of a metallic component for the excellent mechanical behavior of this ceramic. In Japan, zirconia ceramic for TKA has been using since 2001 (KU type, Kyocera Corp, Japan). ${ }^{24}$ The two TKA systems currently manufactured by Kyocera Low Friction Anatomic and Bi-Surface share these features. However, these implants are available for clinical use only in Japan. In Europe, there were several attempts to develop a ceramic TKA. An experimental TKA was developed during the second half of the 1990s in the framework of an European Commission-funded project participated by companies Morgan Matroc (now Morgan Technical Ceramics, Corby, United Kingdom) and LIMA LTO (now Lima Corporate, Villanova di San Daniele del Friuli, Italy). The femoral component of this implant was 
made up of Ti alloy with two zirconia inserts and had a design similar to that of a metallic implant previously brazed on it. ${ }^{25}$ However, the results of this project were never transferred to production. Another company (St Gobain Advanced Ceramics Desmarquest, SGCAD, Evreux, France) developed a zirconia (Y-TZP) TKA. ${ }^{26}$ Clinical tests were performed in Europe ${ }^{27}$ and in the United States, ${ }^{28}$ but the development of this device was abandoned.

Payten and Ben Nissan ${ }^{29}$ developed a femoral component with a metallic baseplate on the magnesia-partially stabilized zirconia condyles were clipped, albeit no further mention of this device can be found in the literature.

The abandonment of all those projects may depend on several reasons. Certainly, giving up SGCAD after the failure of zirconia femoral heads ${ }^{30}$ was one of them. In addition, development of TKA femoral components made up of alumina-matrix composite (BIOLOXdelta; CeramTec) ${ }^{31}$ may have contributed to the dismissal of previous projects.

So far, BIOLOXdelta components are used in two TKA systems with different design: the Multigen Plus (Lima Corporate) and the BPK-S (Peter Brehm, Weisendorf, Germany) (-Fig. 1). The Multigen Plus TKA (available for clinical use) has a ceramic femoral component articulating on a monoblock XLPE tibial component that replaced the metallic tibial tray and XLPE insert of the early design. In the BPK-S, both the femoral and the tibial components are made up of BIOLOXdelta and the ceramic tibial tray hosts a semiconstrained XLPE insert, having a stump in its inferior part articulating with the tray to control its motion. The clinical tests on BPK-S are still in progress.

\section{Literature Results}

Bergschmidt et $\mathrm{al}^{32}$ investigated the results of 62 TKAs 24 months postoperatively. In one group of patients, a symmetric ceramic BIOLOXdelta femoral component (Multigen Plus) was used. Both the femoral and tibial components in all the implants were cemented, while patella was never replaced. The authors observed early nonprogressive asymptomatic radiolucent lines (of up to $1 \mathrm{~mm}$ ) around the tibial component; Short Form 36 (SF-36) score improved significantly only in patients treated with the ceramic femoral component. They

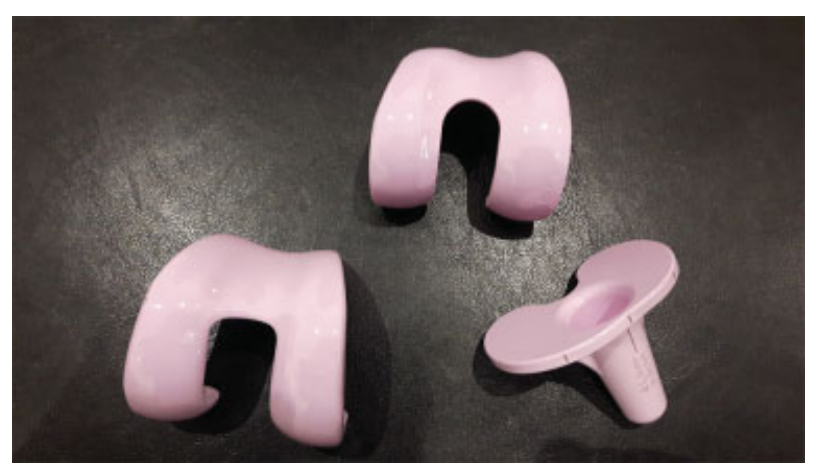

Fig. 1 BIOLOXdelta components for TKA. Lower left: femoral component of the Multigen Plus TKA (Lima Corporate). Upper right and lower right: femoral component and tibial tray of the BPK-S TKA (Peter Brehm). TKA, total knee arthroplasty. concluded that ceramic femoral components performed comparably to traditional metallic designs. ${ }^{32}$ Clinical and radiological results of 109 knees operated with the same implant were subsequently evaluated in a multicenter study at a 5-year follow-up. ${ }^{33}$ Significant improvement in Western Ontario and McMaster Universities Arthritis Index and SF-36 scores were reported, with the implant rated as "excellent" or "good" in $94 \%$ of the cases. Neither implant migration nor loosening was registered; a midline longitudinal crack on the ceramic femoral component as consequence of a patient traumatic fall was reported as isolated adverse event. The overall Kaplan-Meier's survivorship was $96.0 \%$ at 60 months.

Zietz et al $^{34}$ showed that wear of polyethylene inserts under third-body (i.e., bone cement) wear conditions is significantly lower with ceramic femoral components than with metallic femoral components. Previously, Oonishi et $\mathrm{al}^{35}$ compared the wear properties of the combinations of 6 -mm-thick ultrahigh-molecular-weight polyethylene (UHMWPE) insert with Co-Cr alloy, alumina, or TZP ceramic femoral components by using a six-station knee joint simulator test and showed that the wear of the UHMWPE inserts was remarkably lower against the ceramic femoral component than against the $\mathrm{Co}-\mathrm{Cr}$ femoral component. Furthermore, after long-term cycling ceramic, femoral components are not more prone to mechanical loosening than metal ones, and thus mechanical loosening should not be considered as a cause of concern. ${ }^{36}$

Nakamura et $\mathrm{al}^{37}$ reported on the tricompartmental Bi-Surface Knee System (Kyocera), which has a unique balland-socket joint and whose femoral component is made from alumina ceramic. All components-the tibia being made of titanium alloy and the patella as a spherical dome made of UHMWPE with a four-cornered alumina ceramic peg -were fixed with bone cement. The authors showed excellent clinical results, with a survivorship at 10 years of $97.4 \%$ when revision of any component was considered as the end point. Recently, the same authors revised their series at 15-year follow-up and reported survivorship of $94 \%$. Infections and instability accounted for $50 \%$ of the revisions (12 out of 24), while revisions due to fracture of the alumina femoral components and to wear of the UHMWPE insert took place only in 3 out of 24 cases. $^{38}$ Similar outcomes were reported by Nakamura et al, ${ }^{39}$ who reviewed at a minimum follow-up of 10 years (range, 10-13), a cohort of 51 patients with 70 Medial Pivot Knee TKAs (Kyocera). These implants had an alumina femoral component and a titanium alloy tibial component hosting an EtO-sterilized UHMWPE insert. The Kaplan-Meier's survivorship taking revision surgery as end point was $98.6 \%$.

Meier et $\mathrm{al}^{40}$ reported the results of 38 primary metalfree BPK-S ceramic TKAs (Peter Brehm GmbH, Weisendorf, Germany). All the implants were cemented and without patella replacement. Rotating or fixed-bearing inserts were used. Postoperative radiographs adequately corresponded to the preoperative planning. There were no implant failures during the follow-up period; nonprogressive radiolucent lines were seen in one femoral component and five tibial components. The authors concluded that metal-free ceramic TKAs proved to be safe and clinically efficient. ${ }^{40}$ 


\section{Conclusion}

Improvements in stability of the metallic materials in prosthetic joints have been made over the past years. Nevertheless, some individuals develop nonspecific inflammatory reactions related to implant components. Even if there is no clear consensus concerning metal hypersensitivity screening, patients with suspected hypersensitivity should have a dermatologic consultation and should undergo patch testing and lymphocyte transformation testing. Such tests, despite their still questionable diagnostic accuracy, presented to be useful for preventive evaluation of immune reactivity in patients undergoing primary joint replacement surgery and for clinical monitoring of the possible onset of a metal sensitization in patients with implanted devices.

It should be noted that the large majority of the literature data are related to implants using alumina. The use of this ceramic material in arthroplasty recently decreased and ceramic composites are preferred, which outperform alumina in terms of strength, toughness, and scratch resistance. Studies on hip replacement showed great efficacy of composite ceramics in comparison with past generation implants in terms of increased survivorship and minimum wear even in young and high-demanding patients. ${ }^{41,42}$ However, we do not know if these results might be predictive of the performances achievable with composite ceramics in TKA. This should draw attention of the orthopaedic researchers and surgeons on the use of those technologies in TKA implants, for the benefit of our patients in the years to come.

\section{References}

1 AA.VV. RIAP. Registro Italiano Artroprotesi. Third Report. Roma: Pensiero Scientifico; 2016

2 AA.VV. Servizio Sanitario regionale Emilia-Romagna. Rendiconto Attività RIPO 2013: Registro Regionale di Implantologia Protesica Ortopedica. Available at: https://ripo.cineca.it

3 Spencer BA, Cherian JJ, Margetas G, et al. Patellar resurfacing versus circumferential denervation of the patella in total knee arthroplasty. Orthopedics 2016;39(05):e1019-e1023

4 Jiang C, Liu Z, Wang Y, Bian Y, Feng B, Weng X. Posterior cruciate ligament retention versus posterior stabilization for total knee arthroplasty: a meta-analysis. PLoS One 2016;11(01):e0147865

5 Schnaser EA, Elpers ME, Koch CN, Haas SB, Westrich GH, Wright TM. Posterior stabilized polyethylene inserts in total knee arthroplasty: a retrieval study comparing conventional to highflexion designs. J Arthroplasty 2016;31(02):495-500

6 Solarino G, Spinarelli A, Carrozzo M, Piazzolla A, Vicenti G, Moretti B. Long-term outcome of low contact stress total knee arthroplasty with different mobile bearing designs. Joints 2014;2(03):109-114

7 Poirier N, Graf P, Dubrana F. Mobile-bearing versus fixed-bearing total knee implants. Results of a series of 100 randomised cases after 9 years follow-up. Orthop Traumatol Surg Res 2015;101 (4, Suppl):S187-S192

8 Flament EM, Berend KR, Hurst JM, Morris MJ, Adams JB, Lombardi AV Jr. Early experience with vitamin E antioxidant-infused highly cross-linked polyethylene inserts in primary total knee arthroplasty. Surg Technol Int 2016;XXIX:334-340

9 Park DH, Leong J, Palmer SJ. Total knee arthroplasty with an oxidised zirconium femoral component: a 5-year follow-up study. J Orthop Surg (Hong Kong) 2014;22(01):75-79
10 Oonishi H, Kim S-C, Oonishi H, et al. Comparison of in-vivo wear between polyethylene inserts articulating against ceramic and cobalt chrome femoral component in total knee prostheses. In: Chang J-D, Billau K, eds. Bioceramics and Alternative Bearings in Joint Arthroplasty. Darmstadt: Steinkoppf; 2007:149-159

11 Teo WZ, Schalock PC. Metal Hypersensitivity Reactions to Orthopedic Implants. Dermatol Ther 2017;7(01):53-64

12 Kretzer JP, Reinders J, Sonntag R, et al. Wear in total knee arthroplasty-just a question of polyethylene? Metal ion release in total knee arthroplasty. Int Orthop 2014;38(02):335-340

13 Australian Orthopaedic Association. National Joint Replacement Registry. Annual Report. AOA, Adelaide. Available at: https:// aoanjrr.sahmri.com/annual-reports-2016

14 Middleton S, Toms A. Allergy in total knee arthroplasty: a review of the facts. Bone Joint J 2016;98-B(04):437-441

15 Ajwani SH, Charalambous CP. Availability of total knee arthroplasty implants for metal hypersensitivity patients. Knee Surg Relat Res 2016;28(04):312-318

16 Piconi C, Maccauro G. Perspective and trends on bioceramics in joint replacements. In: Antoniac I, ed. Handbook of Bioceramics and Biocomposites. Vol. 2. Berlin: Springer; 2016:821-858

17 Piconi C, Porporati AA, Streicher RM. Ceramics in THR bearings: behavior under off-normal conditions. Key Eng Mater 2015; 631:3-7

18 Eynon-Lewis NJ, Ferry D, Pearse MF. Themistocles Gluck: an unrecognised genius. BMJ 1992;305(6868):1534-1536

19 Lang H. Tibialplateauprothesen aus Aluminiumoxid - Indikation und Egebnisse. Hermsdorfer Technische Mitteilungen 1983;23:1974-1975

20 Langer G. Ceramic tibial plateau of the 70s. In: Garino JP, Willmann G, eds. Bioceramics in Joint Arthroplasty. Stuttgart: Thieme; 2002: $128-130$

21 Oonishi H, Oonishi H, Kim SC. Ceramic knee arthroplasty: advanced clinical experiences of 26 years. Semin Arthroplasty 2006;17:134-140

22 Inoue H, Yakoyama Y, Tanabe G. Follow-up study of alumina ceramic knee (KC-1 type) replacement. In: Oonishi H, Aoki H, Sawai K, eds. Bioceramics. Vol. 3. Tokio: Ishiyaku EuroAmerica; 1989:302-307

23 Lee M-C, Han J-W. Ceramic femoral prosthesis in TKA: present and future. In: Chang J-D, Billau K, eds. Bioceramics and Alternative Bearings in Joint Arthroplasty. Darmstadt: Steinkoppf; 2007:123-132

24 Nakamura T, Oonishi E, Yasuda T, Nakagawa Y. A new knee prosthesis with Bi-Surface femoral component made of zirconia ceramic. Key Eng Mater 2004;254/256:607-609

25 Murray MSG, Facchini A, Sicking R, et al. Development of an advanced ceramic/titanium alloy knee joint. In: Ohgushi $\mathrm{H}$, Hastings GW, Yoshikawa T, eds. Bioceramics. Vol. 12. Singapore: World Scientific Publishing; 1999:67-70

26 Blaise L, Webb J, Calés B. Mechanical analysis of a knee prosthesis with a zirconia femoral component. Orthop Proc 2002;84-B:14

27 Vavrík P, Landor I, Denk F. Clinical evaluation of the ceramic femoral component used for reconstruction of total knee replacement [in Czech]. Acta Chir Orthop Traumatol Cech 2008;75(06):436-442

28 Bal BS, Greenberg DD, Aleto TJ. Primary total knee replacement with a zirconia ceramic femoral component. In: D'Antonio JA, Dietrich M, eds. Bioceramics and Alternative Bearings in Joint Arthroplasty. Darmstadt: Steinkoppf; 2005:83-190

29 Payten WM, Ben Nissan B. Development of a modular ceramic knee prosthesis. In: Wise D, Trantolo DJ, Lewandrowski K-U, et al., eds. Biomaterials Engineering and Devices: Human Applications. Vol. 2. Totowa: Humana Press; 2000:309-336

30 Piconi C, Maccauro G, Pilloni L, Burger W, Muratori F, Richter HG. On the fracture of a zirconia ball head. J Mater Sci Mater Med 2006;17 (03):289-300

31 Heimke G, Leyen S, Willmann G. Knee arthroplasty: recently developed ceramics offer new solutions. Biomaterials 2002;23 (07):1539-1551

32 Bergschmidt P, Bader R, Kluess D, et al. Total knee replacement system with a ceramic femoral component versus two traditional 
metallic designs: a prospective short-term study. J Orthop Surg (Hong Kong) 2013;21(03):294-299

33 Bergschmidt P, Bader R, Ganzer D, et al. Prospective multi-centre study on a composite ceramic femoral component in total knee arthroplasty: five-year clinical and radiological outcomes. Knee 2015;22(03):186-191

34 Zietz C, Bergschmidt P, Lange R, Mittelmeier W, Bader R. Thirdbody abrasive wear of tibial polyethylene inserts combined with metallic and ceramic femoral components in a knee simulator study. Int J Artif Organs 2013;36(01):47-55

35 Oonishi H, Ueno M, Kim SC, Oonishi H, Iwamoto M, Kyomoto M. Ceramic versus cobalt-chrome femoral components; wear of polyethylene insert in total knee prosthesis. J Arthroplasty 2009;24(03):374-382

36 Cristofolini L, Affatato S, Erani P, Tigani D, Viceconti M. Implant fixation in knee replacement: preliminary in vitro comparison of ceramic and metal cemented femoral components. Knee 2009;16(02):101-108
37 Nakamura S, Kobayashi M, Ito H, Nakamura K, Ueo T, Nakamura T. The Bi-Surface total knee arthroplasty: minimum 10-year followup study. Knee 2010;17(04):274-278

38 Nakamura S, Ito H, Nakamura K, Kuriyama S, Furu M, Matsuda S. Long-term durability of ceramic tri-condylar knee implants: a minimum 15-year follow-up. J Arthroplasty 2017;32(06):1874-1879

39 Nakamura S, Minoda Y, Nakagawa S, et al. Clinical results of alumina medial pivot total knee arthroplasty at a minimum follow-up of 10years. Knee 2017;24(02):434-438

40 Meier E, Gelse K, Trieb K, Pachowsky M, Hennig FF, Mauerer A. First clinical study of a novel complete metal-free ceramic total knee replacement system. J Orthop Surg 2016;11:21

41 Piconi C, Streicher RM. Forty years of ceramic-on-ceramic THR bearings. Semin Arthroplasty 2013;24:188-192

42 Sentuerk U, von Roth P, Perka C. Ceramic on ceramic arthroplasty of the hip: new materials confirm appropriate use in young patients. Bone Joint J 2016;98-B(1, suppl A):14-17 